\title{
An Overview of the Pixel Ware Project at the Oriental Museum, Durham
}

\author{
Andrew Richardson \\ University of Sunderland \\ Design Centre, $\square$ City Campus, $\square$ Chester Road, $\square$ Sunderland. $\square$ SR1 3SD \\ andrew.richardson@sunderland.ac.uk
}

\begin{abstract}
Pixel Ware is an interactive table artwork inspired by the world-renowned collection of Chinese ceramics at the Oriental Museum, Durham. Traditional decorative Chinese pattern and symbol from the Oriental Museum's collection are reworked to create digitally generated surface designs projected on to blank ceramic objects on the table. Visitors are able to interact with each of the digitally projected surface patterns as they add, move, or rotate objects on the table. This paper will present an outline the Pixel Ware project; its conceptual background and technical process, and finishing with a brief evaluation of the project as an interactive artwork in the context of the museum. The use of contemporary digital technology within the context of traditional Chinese ceramic design highlights common concerns and intersections between both ancient and modern processes. By interacting with the digital pattern from the collection via tactile manipulation of physical objects, visitors are encouraged to reassess the role of digital technology in the context of traditional craft, to see links between digital and traditionally created pattern design and to consider the role that technology plays in relation to the crafted object. In discussing the project, the presentation will highlight the significance of the relationship between the artwork and the museum collection, present the role of digital interactive art as a means of enhancing museum visitor experience, and explore the application of digital technology in the context of traditional design collections. By examining the relationship between digital and traditional material, the presentation will highlight the idea of 'making' using programming as a means of creating a type of 'digital decorative' design.
\end{abstract}

Digital Interactive Museum Processing reacTIVision Artwork

\section{INTRODUCTION}

The aim of the Pixel Ware project was to create a new perspective on the Chinese ceramic collection at the Oriental Museum, Durham via the creative application of digital, interactive technologies. Situated within the museum alongside the ceramic collection, the artwork sought to create an engaging interactive environment which placed the design and aesthetic of the Chinese symbols alongside a contemporary, digital environment. This was done in order to inspire a sense of awe for the original artefacts, encourage a fresh view of the original collection and create connections between the technical and aesthetic dexterity of both traditional and computational processes.

The project took the form of a practice-based exploration which aligned the use of the 'material' of code in the context of traditional craft. The application of contemporary digital technology alongside traditional Chinese ceramic objects highlights intersections between both ancient and modern processes: The desire to create work which displays visual and aesthetic control and inventiveness, through technological experimentation can be seen as common to both traditional and digital techniques. The piece brought into focus the concept of 'code as craft', exploring the relationship between digital and traditional material, and has particular interest in the use of code in the context of digital decorative design. The use of hand-written programming code, of 'crafting with code' therefore forms an important conceptual element, key to the direction of the work.

\section{CODE AND CRAFT}

The concept of aligning digital processes with the physical process of making and crafting with 'real' physical material is not without precedence: Malcolm McCullough's 'Abstracting Craft' (1998) is a study outlining how ideas of craftsmanship can apply to making in the digital realm, offering a 
broader view of computer practice within a craft ethos. McCullough sheds light on the wider practice of engaging with the computer on a craft-like level, and draws attention to the idea that the lack of physicality of the computer need not prevent notions of it having a 'material quality' being considered. There are many digital practitioners whose approach supports this idea; rejecting the use of pre-built software in favour of the 'directness' and 'freedom' afforded by the direct use of code as the 'material' for their practice. The work of these 'computational designers' embodies an enthusiastic joy for process; for exploring, pushing boundaries and crafting the computational material (Manovich 2002). For many of these designers, code takes on the nature of a material which can be sculpted into forms which are directly related to the thoughts and wishes of the maker in a way which echoes traditional material:

\begin{abstract}
It can be useful to think of each programming language as a material with unique affordances and constraints ... in the same way that the different woods Pine and Oak "feel" and "look" different.(Reas 2003).
\end{abstract}

In this way, the use of programming, like traditional making, becomes a way of hand-making objects, expressing the unique capabilities of the computational material, embodying a 'spirit of craft' (Nakamura \& Fitzpatrick 2008).

The idea of computation as craft has also found expression through a number of recent, high-profile exhibitions; most notably the British Council's My World: New Subjectivity in Design (British Council Arts 2006), the Lovebytes 'Code:Craft' show (2010), and the Victoria and Albert exhibition Decode: Digital Design Sensations (decode | V\&A 2010). Each of these shows explored and made links between the ideas of material and making in the context of digital and computational work, and featured the work of established names from the computational design arena: Daniel Brown (Brown 2010), Golan Levin (Levin 2009), Casey Reas (Reas 2009).

The use and attitude towards material is therefore an important element in computational design, reflecting the ethos of traditional craft and providing a foundational cornerstone upon which both practices are built. It is within this conceptual context that the Pixel Ware project was developed and produced. Concerns with making, skill, mastery, understanding of material and the 'handmade' which inform both computational practice and traditional design work therefore provide an important contextual backdrop to the conceptual development and process of the Pixel Ware project, in which digital interactive work is created to re-interpret traditional Chinese ceramic design. It is now necessary to consider more closely the process and development of the work itself.

\section{PROCESS}

The process of the Pixel Ware project is one which sought to create a successful integration of the traditional Chinese ceramic designs and motifs into a new computational framework. Rather than creating entirely new designs, the work aims to reanimate elements of the original patterns; integrating them into a computational framework to create an evocative, poetic combination of technology and aesthetics. The following section will provide an overview of the process of project; outlining the means by which the original designs were selected, digitized and integrated into a computational environment to create the final computationally animated, interactive work.

\subsection{Image Selection and Digitization}

The collection of Chinese ceramics at the Oriental Museum, Durham contains a wide variety of ceramic artefacts decorated with a range of motifs which represent a variety of typical Chinese decorative symbols and themes. Special access to the collection was granted by the curators and a selection process was undertaken in order to find the most suitable images to be digitised and used as source imagery for the artwork. In selecting pieces to be digitised, as well as the aesthetic qualities of the object, consideration was also given to designs which offered the greatest potential for computational animation, i.e. how suitable elements of the design would be to re-imagine and re-create as part of a generative computational structure. The final selection featured objects decorated with fish, plant (fig. 1) and dragon images, common motifs within the museum's Chinese collection; these were selected for their aesthetic qualities and because they offered the greatest opportunities for visual exploration and experimentation with different types of computational movement, growth and interaction.

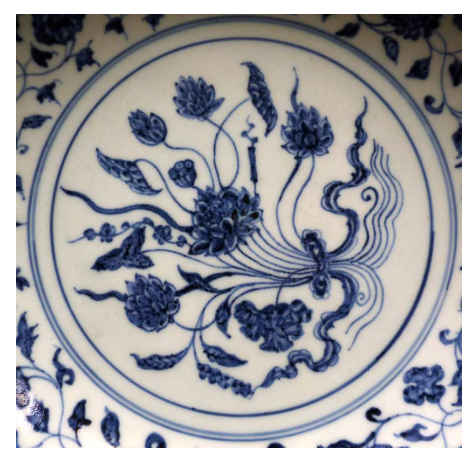

Figure 1: Sample pattern from plate in the collection. 
Once selected, designs from the ceramic objects were digitised for use as source imagery for the artwork. Individual elements or attributes within the original pattern were digitally isolated, allowing them to be used as textures to be applied to the computational shapes and forms (fig. 2). Great care was taken whilst isolating and editing each design so that the inherent individual 'quality' of the shape, mark or brushstroke was maintained, and its original essence preserved. This process was more difficult than first imagined: the 'imperfections' and individuality of the textured quality of the marks, especially of the delicate flowers and stems, were hard to maintain without reducing their aesthetic quality. Also, in complex areas of the design where elements intertwined with others, isolation of individual items became problematic. In these cases digital manipulation and editing was required to preserve the overall aesthetic character of the piece. Once digitized and isolated, the images were used as 'textures' mapped onto computationally created lines and vertex shapes.

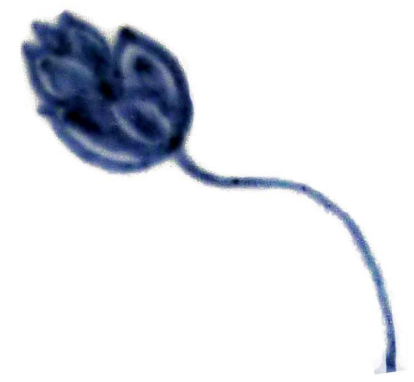

Figure 2: Isolated element from the blue and white design.

\subsection{Texture Mapping}

Texture mapping the bitmap images onto interactive computational objects formed an important part of the work: The key means by which elements of the original designs were integrated into the computational environment; the means of 'bringing to life' and re-animating the original designs of the ceramic pattern.

The process of applying textures to computational objects is not new, and there are many good examples of this practice applied to creative projects (Bang \& Heo 2009). The specific challenge of this project, however, was in maintaining the integrity of the original design and mapping often complex patterns onto a new computational shape. Straight texture shapes with little or no curvature can be mapped directly onto a straight line which can be subsequently computationally manipulated, however a curved texture shape mapped onto a straight computational line distorts wildly and produces unpleasing results. The preservation of the aesthetic integrity of elements from the original design therefore presented a particular difficulty, as they are often complex shapes including many twists and curves. In order to animate the original patterns in a naturalistic way, which preserved the aesthetic form of the original, the textures had to be mapped onto shapes which echoed the shape and curvature of the original pattern.

To solve this important issue, vector lines were 'manually' drawn over the top of each individual image texture using Adobe Illustrator, and exported as an svg file (fig. 3). Vector information of the svg was imported into Processing (Fry \& Reas 2009) via the Geomerative library (Marxer 2010) which allowed access to and retrieval of information about each vector shape.

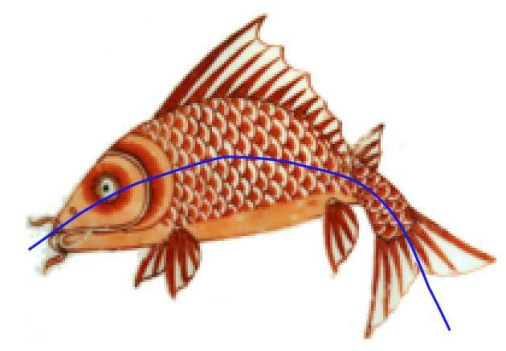

Figure 3: Vector line drawn on top of image texture to 'capture' its shape.

Path information (e.g. points, angles, etc.) was then taken from the imported vector shape and used to created a computational line which matched the shape of the vector, echoing that of the original texture. This allowed the path of the svg vector line to be accurately recreated in Processing and used to create vertex shape onto which the texture was mapped. Decorative textures from the original ceramic patterns were thus successfully mapped onto blank vertex shapes which matched their curvature and form creating computational shapes which maintained the appearance of the original patterns.

\subsection{Adding Behaviour}

The texture mapping process allowed the creation of static computational forms, which retained the shape and aesthetic appearance of elements from the original surface pattern design. The potential to then computationally animate each textured line and shape subsequently allowed the project to experiment with the possibilities of adding a new dimension to the digital shapes; creating patterns which have the ability to be moved, animated, or interacted with, i.e. shapes which have uniquely computational behavioural elements to them. The next part of this discussion will consider the development of these important 'behavioural' elements within the work.

Inspired by close observation of natural forms, traditional decorative pattern often presents a 
'stylised' form of nature done which is not directly copied but stylistically re-presented in an aesthetically controlled and considered manner (Morris 1884). When developing the computationally decorative designs, a similar attitude was adopted, i.e. to embody the spirit of the original designs by applying stylised naturalistic, organic behavioural aspects to each pattern. The computational representation of each pattern theme (plant, fish, dragon) therefore aimed to apply stylised computational behavioural elements of natural form to each element within the design. Algorithmic structures were applied to all of the computational lines giving each a 'behaviour' controlling its growth movement and structure; transforming the static lines and forms into interactive and generative patterns. Individual behaviours were developed and applied to each of the different pattern types, giving each one its own behaviour and style. Consideration of the behavioural element for each pattern is discussed in turn.

\subsubsection{Flower Pattern Behaviour}

The main considerations when developing the aesthetic and behavioural qualities for the plantthemed design, focused upon organic growth, decay and movement: i.e. creating an overall structure which echoed the branching botanical forms of the 'blue and white' designs, whilst embodying a stylised, naturalistic pattern, which possesses 'living' growth and movement. In order to achieve this, a computational structure was created using a pre-defined $2 \mathrm{D}$ array to randomly generate a variety of branching structures. The system allowed a wide variety of random stylised organic plant structures to be created which grow, move and develop in an organic, non-predictable way. The system randomly selects from a set of pre-defined types (branches, leaves, flowers) and generates an organic 'branching' structure of linked vertex shapes. Each individual shape is then mapped with an appropriate texture to define its appearance; the detail of which is subject to further 'random selection' from an array of pre-defined leaf, flower, and branch textures, which further varies the appearance of the details within the pattern.

Growth, development and movement of the branching structure to create an organic, naturalistic, behaviour is achieved by algorithmic manipulation of the points within each individual vertex shape. For example, the gradual expansion of distance between individual points within the vertex shape allows each part of plant shape to gradually 'grow' on the surface of the plate. The application of algorithmic spring forces (Fry \& Reas 2007) applied to the angles of each section of the line creates a springy bounce movement which, when aligned to the speed of movement simulates a stylised organic movement of leaves and 'swaying' branches. Organic decay is added into the system so that shapes and lines gradually fade then disappear to be replaced by other growing organic branching shapes and structures.

\subsubsection{Fish and Dragon Pattern Behaviour}

The structure used for the plant designs was also applied to the fish and dragon shapes. However, without the necessity to generate a variety of complex organic branching structures (i.e. random combinations of branches, leaves and flowers) they were somewhat simpler to realise. Although each of these designs adopted the 'growth' idea from the flower pattern, which allowed the shape to slowly emerge onto the screen, the main focus for both the fish and dragon patterns was centred upon the fluid motion of the shapes themselves. The flexibility of the vertex shape structure allowed individual movements to be created and applied to each individual shape (fig. 4). Variables to control speed, direction, etc. were applied and manipulated to control the individual movement of each object. Flowing, stylised 'swim' movements for each of the fish and dragon forms were generated by algorithmic manipulation of the individual points along each vertex shape, in which sine and perlin noise calculations were added to create a randomised individual motion for each decorative element.

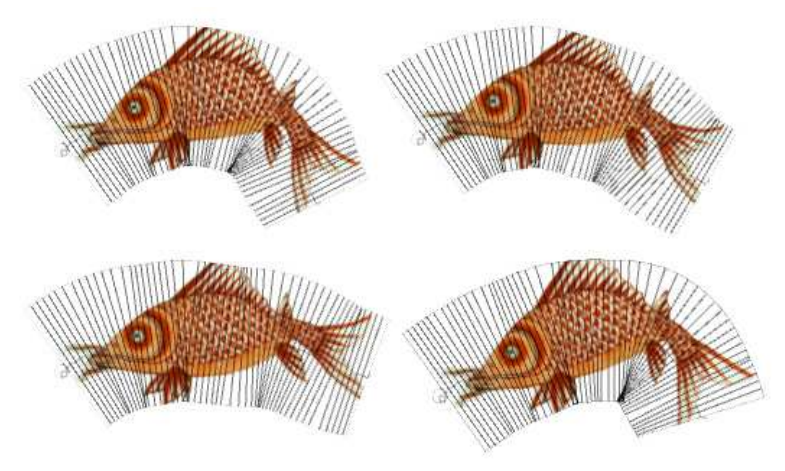

Figure 4: Screenshots of a texture, mapped onto an animated vertex shape.

\subsection{Interactive Table Construction}

The interactive table was designed and built following a standard reacTIVision table set up, (Kaltenbrunner \& Bencina 2010). Infra-red lighting and an infra-red camera were used to highlight and detect movement on the surface of the table. However, unlike the usual reacTIVision table constructions, in which the table top is used as the projection surface, projecting images on to ceramic plates meant that the digital projection had to be positioned above, rather than inside, the table. 


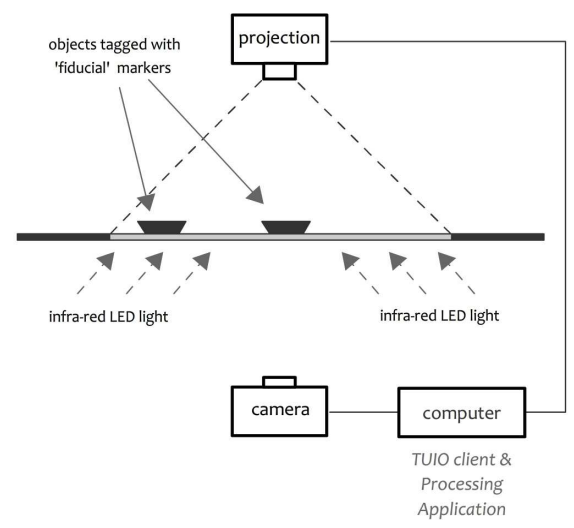

Figure 5: Diagram of the set up.

To achieve this, the original construction design (fig. 5) imagined a projector mounted into the ceiling of the museum, but when this option became unfeasible, an alternative system was designed in which the projector was fixed and directed upwards from within the table structure; the projected image was then reflected onto the table surface via a mirror. This negated the need to physically fix any hardware into the fabric of the building, making the entire set up self-contained and more portable.

'Fiducial' markers attached to the base of each of the plates were used to locate and track each plate's position and movement on the table surface. The computational system assigned a specific set of images and patterns to be created, triggered by individual markers. Each marker therefore triggered a new pattern to be projected on to a plate (fig. 6). Fish, flower and dragon patterns emerged as plates were added onto the table; the patterns organically changing speed and direction in response to the movement of the plate. The final table set-up therefore allowed users to intuitively interact with the computational versions of the original Chinese ceramic designs by playing with the objects on its surface (Richardson 2011).

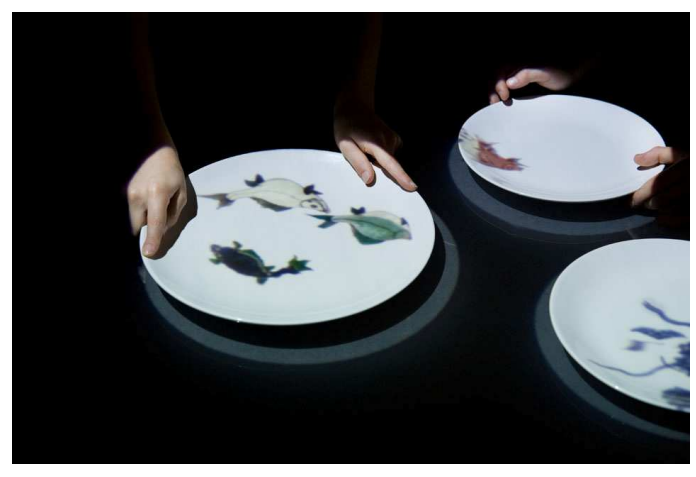

Figure 6: Digital patterns projected onto a plate.

\section{MUSEUM CONTEXT}

The situation of the work in the museum and its relationship to the collection plays an integral part to the understanding, significance and meaning of the artwork. This final section of the paper will present an overarching discussion of the project in terms of the relationship between the artwork in the context of the museum collection.

The Oriental Museum provided an important, influential context for the development of the Pixel Ware project, providing both visual inspiration via physical access to objects within the collection, and conceptual inspiration regarding the relationship between digital and traditional forms of decorative design. The development phase of the project established a relationship between the work and the museum which played a significant role in the aesthetic and conceptual development of the piece. Once completed, the installation of Pixel Ware within the Chinese gallery space further extended this relationship. The physical situation of the artwork alongside the ceramic collection generated an interesting interplay between the original artefacts, and their digital re-interpretations. By situating Pixel Ware directly within the ceramic collection, the meaning and the significance of each changed and developed; both the traditional museum objects and the interactive digital artwork began to reflect, comment on and inform each other, encouraging visitors to generate a fresh understanding and perspective of each.

The Pixel Ware artwork therefore created a new, different 'voice' within the museum (Bradburne 2002) which challenged expectations, encouraged exploration of the collection, and promoted an open-ended interplay between the visitors, the art and its surrounding artefacts. Observation of, and feedback from, visitors revealed how interaction with Pixel Ware informed and stimulated engagement with the wider museum collection. By using the work as a way of re-discovering the designs used in the piece, visitors reflected on the way in which engagement with Pixel Ware 'brought life' to the original collection, stimulating a creative dialogue between the artwork and the wider museum collection. The multi-user nature of the interaction, in which physical objects were manipulated on the table, further encouraged interaction and conversation, as users explored and experimented with the piece, moving or even stacking plates on the table's surface. 


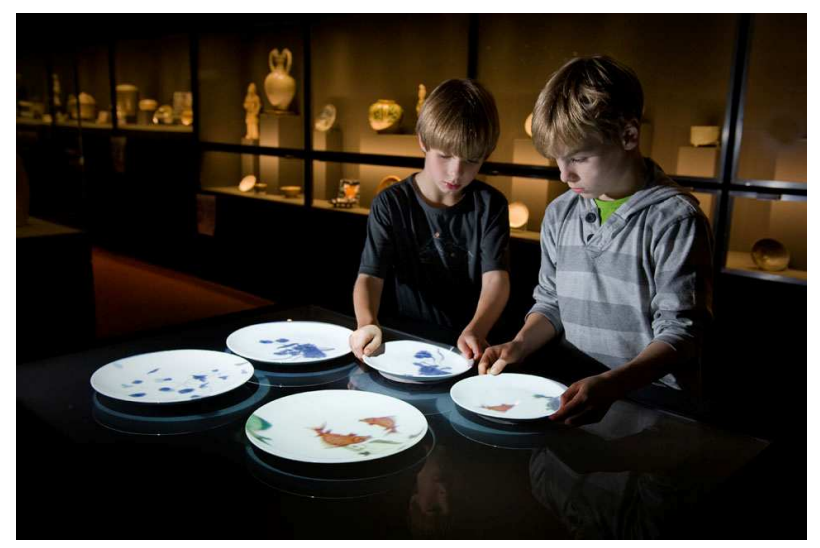

Figure 7: Interacting with the work in the museum.

Visitor interaction with, and use of the work also revealed new connections between the artwork and the museum collection. Observed audience fascination with the magical 'transformation' of the blank white plates into interactive digital surfaces as they were placed on the table, revealed unexpected connections between the digital and traditional objects in the museum collection, bringing new layers of meaning to the work. Many users, intrigued with the effect of adding and moving plates onto the table surface, began to consider these ordinary objects with a special significance. The objects within the interactive, digital environment therefore began, in some small way, to reflect the special ('magical') significance and aura possessed by the objects in the museum. The significance and uniqueness of the physical objects in the collection was echoed by a new significance given to the plates on the table as they underwent their digital transformation. This unexpected demonstration of the 'power' that tangible objects can possess, even in the intangible digital, computational environment, shows that use of the physical object plays an important part in the digital artwork; providing a new, and interesting way for users to interact with, and consider, objects in the collection, and adding a further layer of connection between the artwork and its surroundings. This interplay between the collection, the artwork and the user therefore highlights how the positioning of the artwork alongside the collection creates more complex layers of meaning; challenging elements of traditional and digital art, encouraging visitors to approach view the collection, and digital art, in a new way, revealing unexpected connections between traditional and digital media.

\section{CONCLUSION}

The process and outcome of the Pixel Ware project has revealed several important elements which have been the highlighted in this paper.
The project's use of digital technology, and particularly programming code, in re-animating elements of the museum's collection emphasises intersections between digital and traditional aesthetics and ways of making; creating connections between traditional craft and computational work; and reflecting the underlying concept of code as craft. The transformation of fish, plant and dragon motifs from the Chinese collection, into computationally animated and dynamic surface patterns, highlights the successful application of a specific texture mapping processes; giving organic, algorithmic behavioural qualities to the original designs from the museum.

The nature of the interactive table, with its emphasis on physical objects (plates) as the core method of user exploration, represents a new application of the reacTIVision framework, which works to create a distinctive method of user engagement and which generates connections and layers of meaning between the digital and physical artefacts. The situation of the artwork within the museum environment also creates an interesting interplay between the objects and the interactive plates on the table; encouraging open-ended discussion and discovery by users and visitors as playful interaction with the interactive surface leads to further exploration and investigation into the original collection itself.

It is therefore possible to see how the production and installation of the Pixel Ware project has provided an important and useful means by which themes, relationships and intersections between digital and traditional artworks have been explored and discussed.

\section{REFERENCES}

Bang, H. and Heo, Y. (2009) OASIS. http://everyware.kr/portfolio/contents/09_oasis/ (retrieved June 2011).

Bradburne, J. (2002) Museums and Their Languages: Is Interactivity Different for Fine Art as Opposed to Design? Interactive Learning in Museums of Art and Design, Victoria and Albert Museum, London, UK, 17-19 May 2002, http://media.vam.ac.uk/media/documents/legacy_d ocuments/file_upload/5758_file.pdf.

British Council Arts (2006) My World - crafts and applied arts - British Council Arts. http://www.britishcouncil.org/arts-aad- designcrafts-and-applied-art-my-world.htm (retrieved July 2007).

Brown, D. (2010) Daniel Brown's. http://www.danielbrowns.com/ (retrieved April 2010). 
decode | V\&A. (2010)

http://www.vam.ac.uk/microsites/decode/ (retrieved date).

Fry, B. and Reas, C. (2009) Processing.org. http://processing.org/ (retrieved March 2010).

Fry, B. and Reas, C. (2007) Processing A Programming Handbook for Visual Designers and Artists. MIT Press, Cambridge, MA.

Kaltenbrunner, M. and Bencina, R. (2010) reacTIVision 1.4. http://reactivision.sourceforge.net/ (retrieved January 2011).

Levin, G. (2009) Flong Interactive Art by Golan Levin and Collaborators. http://www.flong.com/ (retrieved February 2010).

Manovich, L. (2002) Generation Flash. http://manovich.net/DOCS/generation_flash.doc (retrieved September 2010).

Marxer, R. (2010) Geomerative. http://www.ricardmarxer.com/geomerative/

(retrieved May 2011).
McCullough, M. (1998) Abstracting Craft: The Practiced Digital Hand. MIT Press, Cambridge, MA.

Morris, W. (1884) Some Hints on PatternDesigning.

http://www.marxists.org/archive/morris/works/1881/ hints.htm (retrieved April 2010).

Nakamura, Y. and Fitzpatrick, M. (2008) Yugo Nakamura: The Craftsman. Creative Review, February 2008 pp.46-48.

Reas, C. (2003) Programming Media. In Stocker H., \& Schopf C. (eds). Code - The Language of Our Time: Ars Electronica 2003, Hatje Cantz, Osterfildern-Ruit.

Reas, C. (2009) REAS.com / Casey Reas. http://reas.com (retrieved May 2010).

Richardson, A. (2011) Pixel Ware | Andrew Richardson: Computational Design http://agrichardson.com/?portfolio=pixel-ware (retrieved September 2011). 